\title{
Research on multidimensional loading device of material mechanical test
}

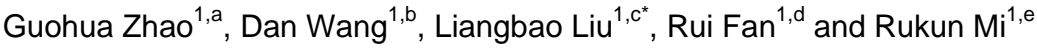 \\ ${ }^{1}$ School of Mechanical Engineering and Automation, Beihang University, 100191 Beijing, China
}

\begin{abstract}
A multidimensional loading device for the material mechanical test based on Stewart Platform was proposed and manufactured in this paper. A determination method of mechanism parameters toward specific engineering requirements was analysed via inverse kinematics and contact interference boundary condition of mechanism components. A set of optimized mechanism parameters was achieved and then the pose space was obtained. Meanwhile, five repeated quasi-static standard tensile tests were performed on the multidimensional loading device and the standard tensile testing machine, respectively. The average yield strength and ultimate strength of the specimen achieved from the different testers were both merely $1.13 \%$. Results indicated that material mechanical multidimensional loading could be conducted by the parallel mechanism.
\end{abstract}

\section{Introduction}

To guarantee engineering structure or machinery working in order, components should possess enough capacity to bear the loads on them [1]. Mechanics of material mainly focuses on deformation, force, and failure analysis. It provides related theory for optimizing component design, such as strength, stiffness, and stability [2]. Majority of formulas in mechanics of material were derived from mechanical tests and were established with many hypotheses and simplifications. Therefore, errors were inevitably generated and may deeply affect the validity and accuracy of the theoretical calculation. Yield strength derived by Von Miss were $15 \%$ greater than that obtained by Tresca in the pure torsional experiment [3]. Furthermore, many failure analyses, in practical application, could not be conducted by theoretical calculation [4]. Consequently, to pursue more accurate mechanical properties and the effective utilization of materials, a considerable amount of different material mechanical experiments were done. In addition to standard single axis tests, two-step uniaxial tests [5], plane biaxial tests [6], combined tension/torsion strain path change tests [7-8] were carried out. However, multidimensional loading experiments that are more in line with the practical engineering application were rarely performed. The main reason that these experiments were not conducted was the lack of multidimensional loading device $(M L D)$ [9].

In this work, a $M L D$ for material mechanical test based on Stewart Platform was proposed and manufactured. A brief architecture description and the inverse kinematics of the $M L D$ were presented at the beginning and then the detailed method of designing and optimizing mechanism parameters were given. Finally,

\footnotetext{
${ }^{\mathrm{c}}$ Corresponding author: 1lb1985101@163.com
}

five repeated standard quasi-static tensile experiments were conducted on the $M L D$ and Zwick Z100, respectively. Results indicated that it was feasible to perform material mechanical experiments on the $M L D$.

\section{Architecture description of the MLD}

The prototype of the $M L D$, shown in fig. 1, was composed of a fixed base, a mobile platform, and six identical motor-driven kinematic chains. Each limb, possessing a ball screw prismatic joint, connected the base with a Hook hinge and a spherical joint to the mobile platform. Fig. 2 was the schematic diagram of the $M L D$. A fixed Cartesian frame (Op-XYZ) and a moving Cartesian frame (Ob-xyz) were settled at the center of the base and the mobile platform, see fig. 2(a). According to the top view, see fig. 2(b), the coordinates of the Hook hinge center $\mathbf{P}_{i}(i=1 \sim 6)$ were denoted by eq.(2), while

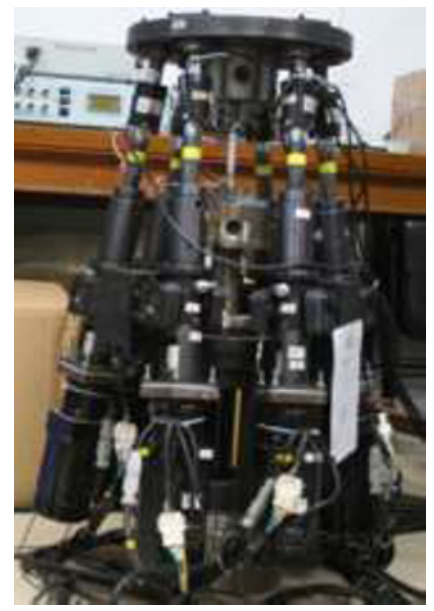

Figure 1. Prototype of the $M L D$. 


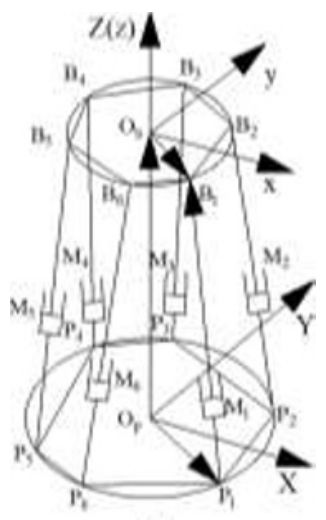

(a)

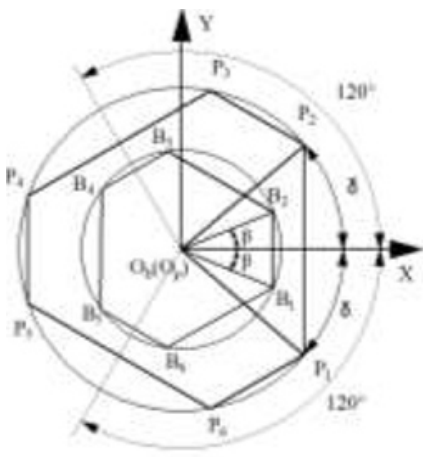

(b)
Figure 2. Schematic diagram of the $M L D$.

eq.(1) described the coordinates of the spherical joint center $\mathbf{B}_{i}(i=1 \sim 6)$.

$$
\begin{aligned}
& \mathbf{B}_{b 1}=[r \cos (b), r \sin (-b), 0] \\
& \mathbf{B}_{b 2}=[r \cos (b), r \sin (b), 0] \\
& \mathbf{B}_{b 3}=[r \cos (2 p / 3-b), r \sin (2 p / 3-b), 0] \\
& \mathbf{B}_{b 4}=[r \cos (2 p / 3+b), r \sin (2 p / 3+b), 0] \\
& \mathbf{B}_{b 5}=[r \cos (4 p / 3-b), r \sin (4 p / 3-b), 0] \\
& \mathbf{B}_{b 6}=[r \cos (4 p / 3+b), r \sin (4 p / 3+b), 0] \\
& \mathbf{P}_{1}=[R \cos (d), R \sin (-d), 0] \\
& \mathbf{P}_{2}=[R \cos (d), R \sin (d), 0] \\
& \mathbf{P}_{3}=[R \cos (2 p / 3-d), R \sin (2 p / 3-d), 0] \\
& \mathbf{P}_{4}=[R \cos (2 p / 3+d), R \sin (2 p / 3+d), 0] \\
& \mathbf{P}_{5}=[R \cos (4 p / 3-d), R \sin (4 p / 3-d), 0] \\
& \mathbf{P}_{6}=[R \cos (4 p / 3+d), R \sin (4 p / 3+d), 0]
\end{aligned}
$$

\section{Inverse kinematics analysis}

To make the description of the moving Cartesian frame's pose, which was expressed in the fixed Cartesian frame, consistent with the rotational motion along axes of the fixed Cartesian frame, the $R P Y$ rotational matrix was selected, see eq.(3).

Where, $c=\cos , s=\sin . \varphi 1, \varphi 2$ and $\varphi 3$ were rotational angles of the moving Cartesian frame corresponding to the $\mathrm{X}, \mathrm{Y}$ and $\mathrm{Z}$ axes, respectively.

$$
\mathbf{R}=\left[\begin{array}{ccc}
c \varphi_{1} c \varphi_{2} & c \varphi_{1} s \varphi_{2} s \varphi_{3}-s \varphi_{1} c \varphi_{3} & c \varphi_{1} s \varphi_{2} c \varphi_{3}+s \varphi_{1} s \varphi_{3} \\
s \varphi_{1} c \varphi_{2} & s \varphi_{1} s \varphi_{2} s \varphi_{3}-c \varphi_{1} c \varphi_{3} & s \varphi_{1} s \varphi_{2} c \varphi_{3}+c \varphi_{1} s \varphi_{3} \\
-s \varphi_{2} & c \varphi_{2} s \varphi_{3} & c \varphi_{2} c \varphi_{3}
\end{array}\right]
$$

The inverse kinematics of this $M L D$ was the solution of the limbs' length $\left(\left|\mathbf{P}_{i} \mathbf{B}_{i}\right|\right)$ based on the mobile platform' pose. Take any limb, in fig. 2(a), as a research object. According to the vector algebra, eq.(4) could be expressed as below.

$$
\begin{array}{ll}
\mathbf{P}_{i} \mathbf{B}_{i}=\mathbf{O}_{p} \mathbf{O}_{b}+\mathbf{O}_{b} \mathbf{B}_{i}-\mathbf{O}_{p} \mathbf{P}_{i} & (i=1 \sim 6) \\
\mathbf{B}_{p i}=\mathbf{R} \mathbf{B}_{b i}+\mathbf{O}_{p} \mathbf{O}_{p b} & (i=1 \sim 6)
\end{array}
$$

Substituting eq.(4) into eq.(5), the vector equation of the inverse kinematics was given bellow.

$$
\mathbf{P}_{i} \mathbf{B}_{p i}=\mathbf{O}_{p} \mathbf{O}_{p b}+\mathbf{O}_{p b} \mathbf{B}_{p i}-\mathbf{O}_{p} \mathbf{P}_{i} \quad(i=1 \sim 6)
$$

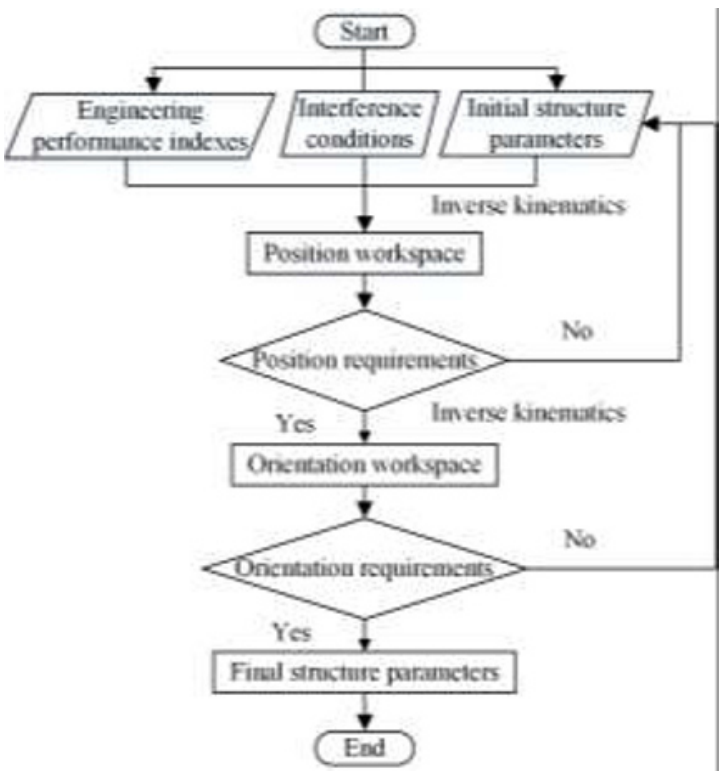

Figure 3. Two-step method of designing the $M L D$ parameters.

\subsection{Determination of the MLD structure parameters}

The workspace of parallel mechanism including the position and orientation spaces was used to determine the structure parameters. Considering position and orientation simultaneously could result in a considerable calculation, and make it impossible to intuitively describe the pose space. A two-step method of the pose solution was introduced to design the $M L D$ parameters. Firstly, selected a set of initial structure parameters based on the engineering performance indexes, and determined the interference conditions of the parallel mechanism. Secondly, using the inverse kinematics to obtain the position workspace based on the previous results; unless the initial parameters could meet the requirements of the engineering indexes, the program went down to the next step or went back. Thirdly, selected a subspace from the position workspace to verify whether or not the orientation workspace could meet the engineering indexes; the final structure parameters was obtained only if the orientation conditions was met. Detailed process of the two-step method was described in fig. 3 .

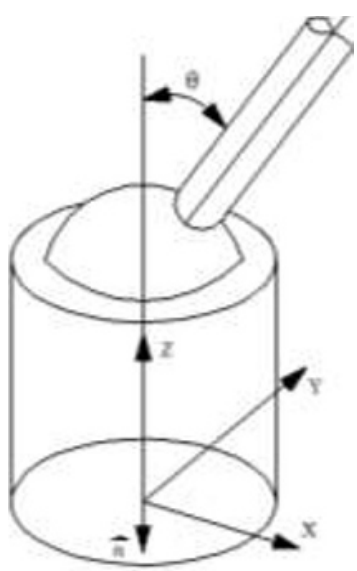

Figure 4. Spherical joint. 


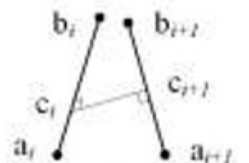

(a)

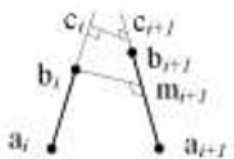

(c)

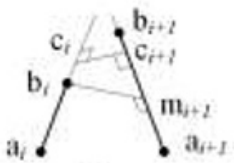

(b)

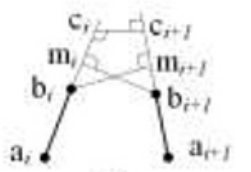

(d)
Figure 5. Four situations of two spatial lines.

\subsubsection{Contact interference boundary conditions}

Contact interference conditions of the mentioned $M L D$ were composed of three kinds of interference conditions: the angle range of joints, limbs' length range, and contact interference conditions of components.

According to fig. 4, the spherical joint interference condition could be expressed by eq.(7). Note here that vector $\mathbf{n}$ denoted the unit vector along the $\mathrm{z}$ axis of the mobile Cartesian frame.

$$
\theta_{p i}=\arccos \frac{\mathbf{B}_{i} \mathbf{P}_{i} \mathrm{~g}\left(\mathbf{T} \mathbf{n}_{p i}\right)}{\left|\mathbf{B}_{i} \mathbf{P}_{i}\right|} \leq \theta_{\max } \quad(i=1 \sim 6)
$$

Similar to the spherical joint, the interference conditions of the Hook hinge were given by eq.(8).

$$
\gamma_{p i}=\arccos \frac{\mathbf{B}_{\mathrm{pi}} \mathbf{P}_{i} \mathbf{g}\left(\mathbf{T} \mathbf{N}_{p i}\right)}{\left|\mathbf{B}_{p i} \mathbf{P}_{i}\right|} \leq \gamma_{\max } \quad(i=1 \sim 6)
$$

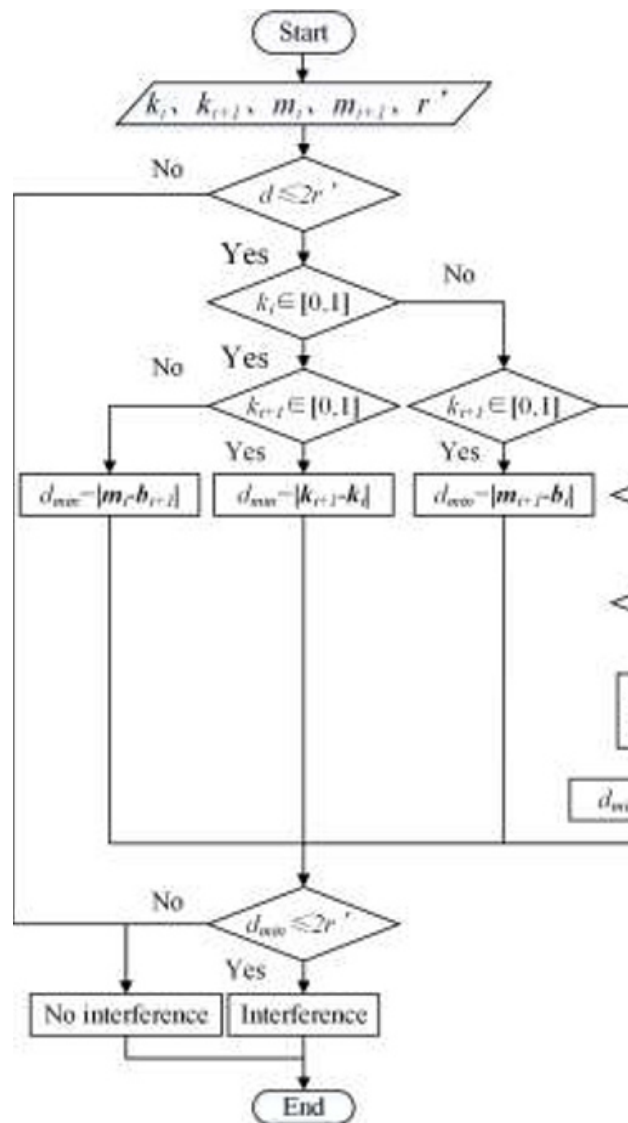

Where $\mathbf{N}_{p i}$ represented the unit vector along the connecting line of two adjacent Hook hinges' rotary center, see fig. 1 .

Each length range of limbs could be calculated from eq.(9), as bellow.

$$
l_{\min } \leq\left|l_{i}\right| \leq l_{\max } \quad(i=1 \sim 6)
$$

According to the structure of the $M L D$, the contact interferences only could occur at the end of the motors or at positions where limbs were close to the upper grip. Since the principle of these two kinds of interferences was the same, only the interferences at the end of the motors were analysed in this paper. To simplify the interference analysis, assuming that the motor was a cylinder whose radius was $r$. Therefore, the contact interference analysis between two motors was transferred to the problem of the contact of two cylinders. Let dmin be the minimum distance between two cylinders, $d$ be the length of the common normal line of these two cylinders' centreline, shown in fig. 5. The radius of the circumscribed circle of the motor, denoted by $r_{l}$, could be obtained by eq. (10). Let $s$ be the side length of the motor and $r^{\prime}=r_{l}$. If the thick line segment $a_{i} b_{i}$ and $a_{i+1} b_{i+1}$, shown in fig. 5 , did not contact with each other, adjacent motors would not occur contact interference.

$$
r_{1}=\sqrt{2} s / 2
$$

It could be divided into two main situations according to the relative position of the two thick lines: (1) If $d>$ $2 r^{\prime}$, motors would not contact each other; (2) if $d \leqslant 2 r^{\prime}$, contact interference could be subdivided into four cases, shown in fig. 5. Detailed analysis of the cases, in fig. 6, were summarized:

Figure 6. Flow chart of the analysis of contact interference. 
1) $d \min =d$, for case fig. 5(a);

2) $d \min =b_{i} m_{i+l}$, for case fig. 5(b);

3) $d \min =b_{i} m_{i+1}$, for case fig. 5(c);

4) $d \min =b_{i} b_{i+1}$, for case fig. $5(\mathrm{~d})$.

The common normal line $\left(c_{i} c_{i+1}\right)$ of line segment $a_{i} b_{i}$ and $a_{i+1} b_{i+1}$ could be expressed by eq.(11).

$d=\left|\left(\mathbf{b}_{i}-\mathbf{b}_{i+1}\right) \frac{\left(\mathbf{b}_{i}-\mathbf{a}_{i}\right) \times\left(\mathbf{b}_{i+1}-\mathbf{a}_{i+1}\right)}{\left\|\left(\mathbf{b}_{i}-\mathbf{a}_{i}\right) \times\left(\mathbf{b}_{i+1}-\mathbf{a}_{i+1}\right)\right\|}\right| \quad(i=1 \sim 6)$

Where $\boldsymbol{a}_{i}, \boldsymbol{b}_{i}, \boldsymbol{a}_{i+1}$ and $\boldsymbol{b}_{i+1}$ were the vectors described in the fix Cartesian frame.

Let $\mathbf{m}$ be the vector along $\boldsymbol{a}_{i} \boldsymbol{b}_{i}$. It could be expressed by the parametric equation, see eq.(12). Meanwhile, the feet of perpendicular $c_{i}$ and $c_{i+1}$ could be obtained by solving eq.(13).

$$
\begin{gathered}
\mathbf{m}_{i}=\mathbf{a}_{i}+k_{i}\left(\mathbf{b}_{i}-\mathbf{a}_{i}\right) \quad(i=1 \sim 6) \\
\left\{\begin{array}{c}
{\left[\mathbf{a}_{i}+k_{i}\left(\mathbf{b}_{i}-\mathbf{a}_{i}\right)-\mathbf{a}_{i+1}-k_{i+1}\left(\mathbf{b}_{i+1}-\mathbf{a}_{i+1}\right)\right] \mathrm{g}\left(\mathbf{a}_{i}-\mathbf{b}_{i}\right)=0} \\
{\left[\mathbf{a}_{i}+k_{i}\left(\mathbf{b}_{i}-\mathbf{a}_{i}\right)-\mathbf{a}_{i+1}-k_{i+1}\left(\mathbf{b}_{i+1}-\mathbf{a}_{i+1}\right)\right] \mathrm{g}\left(\mathbf{a}_{i+1}-\mathbf{b}_{i+1}\right)=0} \\
(i=1 \sim 6)
\end{array}\right.
\end{gathered}
$$

Unless $k_{i} \in[0,1]$, point $m_{i}$ lied between the points $a_{i}$ and $b_{i}$; unless $k_{i+1} \in[0,1]$, point $m_{i+1}$ lied between the points $a_{i+1}$ and $b_{i+1}$. Let $b_{i} M_{i+1}$ be the perpendicular line of $a_{i+1} b_{i+1} . m_{i+1}$ could be derived from eq. (14). Detailed flow chart of the contact interference was shown in fig. 6 .

$$
\begin{array}{r}
{\left[\mathbf{b}_{i}-\mathbf{a}_{i+1}-m_{i+1}\left(\mathbf{b}_{i+1}-\mathbf{a}_{i+1}\right)\right] \mathrm{g}\left(\mathbf{b}_{i+1}-\mathbf{a}_{i+1}\right)=0} \\
(i=1 \sim 6)
\end{array}
$$

\subsubsection{Structure parameters and workplace of MLD}

Based on the inverse kinematics and the interference boundary condition, structure parameters and the position workspace were obtained. Optimized parameters were summarized in table 1; different views of the position workspace were plotted in fig. 7 , respectively. Different from the serial mechanism, the position and orientation workspaces of the parallel mechanism possessed strong coupled effects with each other. Consequently, the pose workspace usually be expressed by specific engineering

Table 1. Structure parameters of the $M L D$.

\begin{tabular}{|c|c|}
\hline Structure parameters & Value \\
\hline $\mathrm{r}[\mathrm{mm}]$ & 120.00 \\
\hline $\mathrm{R}[\mathrm{mm}]$ & 175.86 \\
\hline$\beta\left[^{\circ}\right]$ & 22.50 \\
\hline$\delta\left[^{\circ}\right]$ & 34.46 \\
\hline Initial length of $\mathrm{L}[\mathrm{mm}]$ & 370.00 \\
\hline
\end{tabular}

Table 2. Pose workspace of the $M L D$.

\begin{tabular}{|c|c|}
\hline Pose workspace parameters & Value \\
\hline $\mathrm{X}[\mathrm{mm}]$ & \pm 40 \\
\hline $\mathrm{Y}[\mathrm{mm}]$ & \pm 40 \\
\hline $\mathrm{Z}[\mathrm{mm}]$ & $410 \sim 440$ \\
\hline $\mathrm{X}\left[{ }^{\circ}\right]$ & $-8 \sim 8$ \\
\hline $\mathrm{Y}\left[{ }^{\circ}\right]$ & $-8 \sim 8$ \\
\hline $\mathrm{Z}\left[{ }^{\circ}\right]$ & $-8 \sim 8$ \\
\hline
\end{tabular}

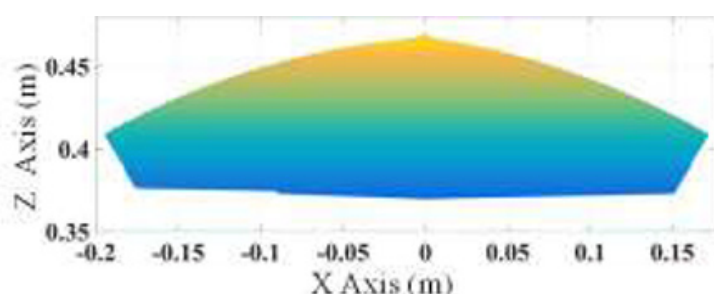

(a)

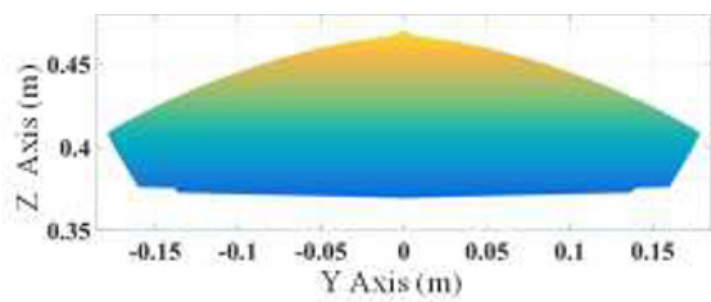

(b)

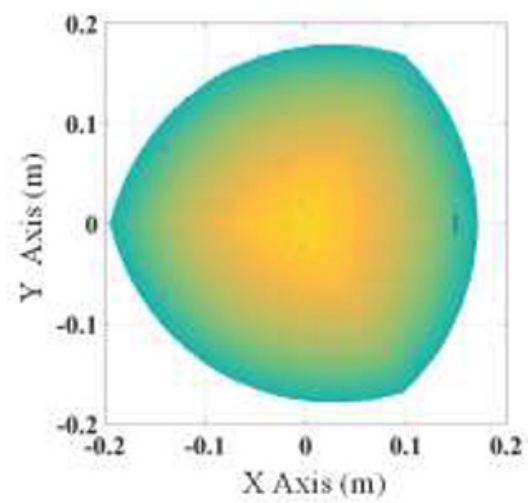

(c)

Figure 7. Different views of the position space.

performance indexes. As for this $M L D$, the pose workspace could be described that the orientation space should be as large as possible under the premise of meeting the requirements of the position space, see table 2.

\section{Material mechanical experiments}

Since the mobile platform of this $M L D$ had six degrees of freedom, it could act more general loads on the material specimen, such as $\mathrm{F}_{x}, \mathrm{~F}_{y}, \mathrm{~F}_{z}, \mathrm{M}_{x}, \mathrm{M}_{y}$, and $\mathrm{M}_{z}$. As for this mechanism, as long as the $M L D$ possessed enough stiffness, strength, motion space, and measurement accuracy, it could be used as a material testing machine. Translations and rotations along the three axes of the fixed Cartesian frame were the same in mechanism theory. Therefore, only tensile tests were performed to verify the loading capacity in this paper. In this work, specimen was manufactured using AISI 1045 steel widely used in practical application. All samples were referenced

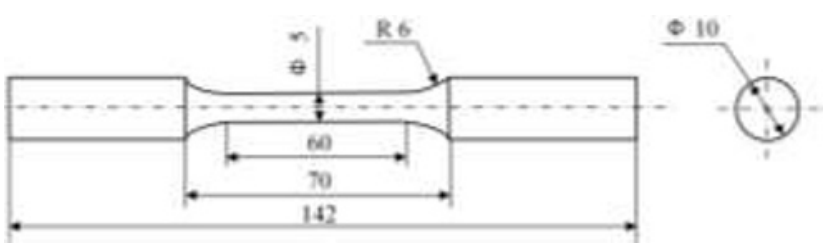

Figure 8. Specimen geometry. 
to the Chinese national standard $G B / T$ 10128-2007, see fig. 8. Chemical composition of the specimen was $97.2 \% \mathrm{Fe}, 0.46 \% \mathrm{C}, 0.24 \% \mathrm{Si}, 0.45 \% \mathrm{Mn}, 0.015 \% \mathrm{Cr}$, $0.08 \% \mathrm{P}, 0.003 \% \mathrm{~S}, \geqslant 0.01 \% \mathrm{Co}$, and $0.01 \% \mathrm{Ni}$. The measured average hardness of these specimen was $209.17 \mathrm{HB}$.

Five repeated tensile tests were conducted on the $M L D$ and the standard tensile testing machine Zwick Z100 according to the Chinese national standard $G B / T$ 10128-2007, respectively. Experimental photos of these two devices were shown in fig. 9, and fig. 10. Stress-train curves obtained from these tests were plotted in fig. 11 and fig. 12, respectively.

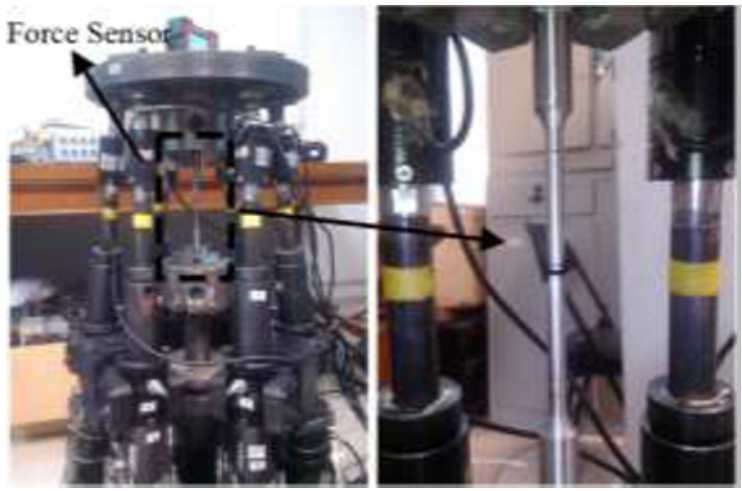

Figure 9. Tensile tests on the $M L D$.

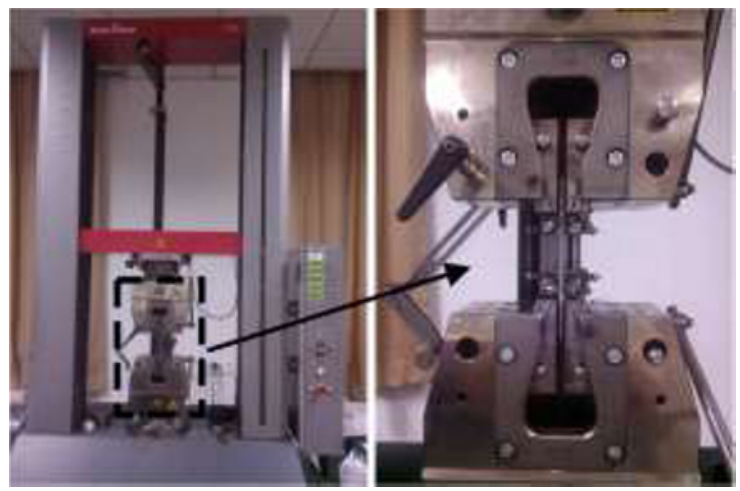

Figure 10. Tensile tests on the Zwick Z100.

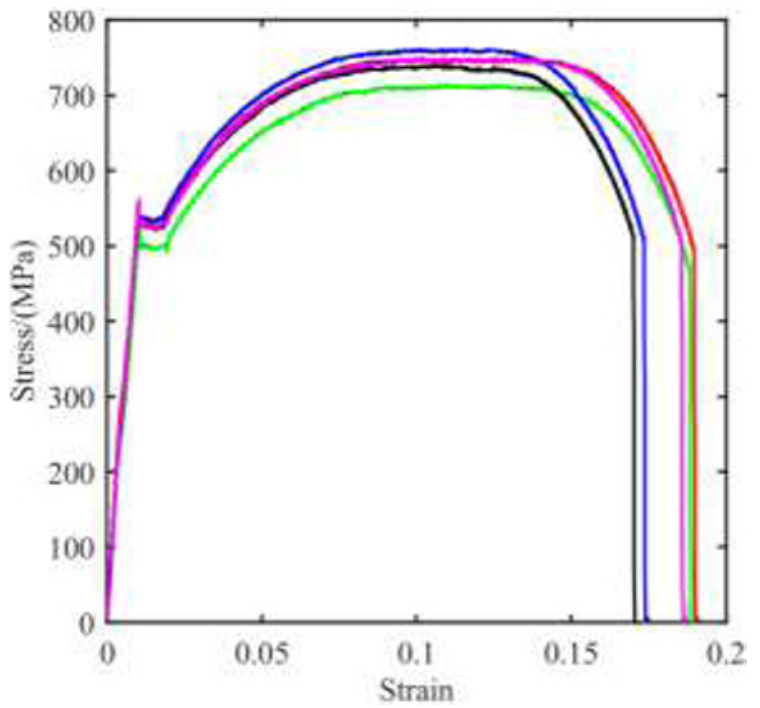

Figure 11. Stress-strain curves based on $M L D$.

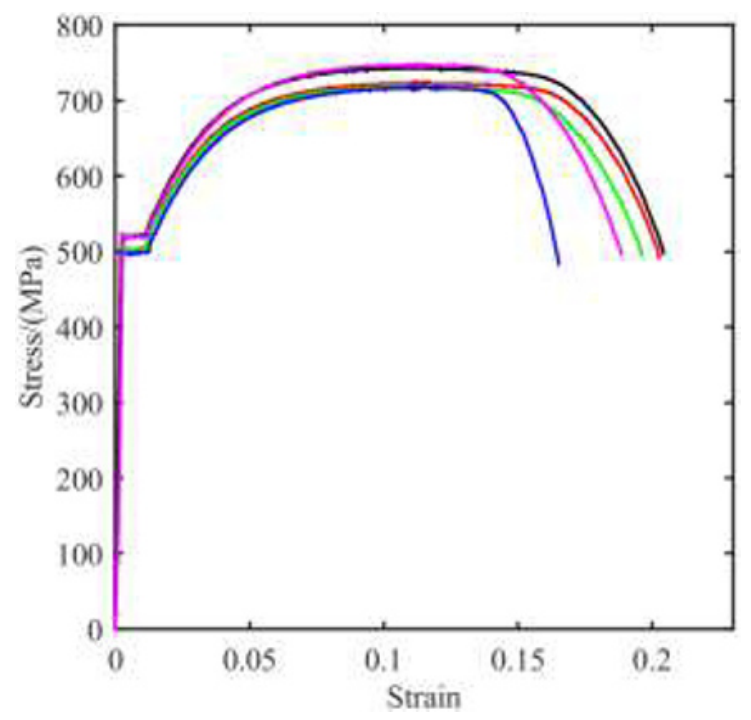

Figure 12. Stress-strain curves based on Zwick Z100.

Table 3. Comparison of mechanical properties derived from $M L D$ and Zwick Z100.

\begin{tabular}{|c|c|c|c|}
\hline $\begin{array}{c}\text { Mechanical } \\
\text { properties }\end{array}$ & $\begin{array}{c}\text { MLD } \\
\text { tests }\end{array}$ & $\begin{array}{c}\text { Zwick Z100 } \\
\text { tests }\end{array}$ & Error [\%] \\
\hline $\begin{array}{c}\text { Average yield } \\
\text { strength } \sigma_{\mathrm{s}}[\mathrm{Mpa}]\end{array}$ & 521.36 & 515.52 & 1.13 \\
\hline $\begin{array}{c}\text { Average ultimate } \\
\text { strength } \sigma_{\mathrm{b}}[\mathrm{Mpa}]\end{array}$ & 737.64 & 729.40 & 1.13 \\
\hline
\end{tabular}

Mechanical properties derived from the two testers were compared and summarized in table 3 .

Errors of the elastic segment in fig. 11 and fig. 12 were caused by that extensometer was not installed on the $M L D$ while Zwick Z100 possessed an extensometer. Meanwhile, table 3 manifested that the maximum errors of $\sigma_{\mathrm{s}}$ and $\sigma_{\mathrm{b}}$ were merely both $1.13 \%$. Therefore, results indicated that it was feasible to conduct material mechanical experiments on the $M L D$.

\section{Summary}

Aiming at the defects of the traditional material testers, on which the more general loads could not be applied, a new material mechanical $M L D$ based on the parallel mechanism was proposed. Meanwhile, pure tensile experiments of AISI 1045 steel were performed on the $M L D$ and on the standard tensile tester Zwick Z100, respectively. Main conclusions could be drawn as follows:

(1) Detailed contact interference boundary conditions were analysed which could be applied to the design of the parallel mechanism;

(2) The method of designing and optimizing structure parameters of the parallel mechanism was proposed based on the inverse kinematics and the contact interference boundary condition;

(3) Errors of the average yield and ultimate strengths obtained from the two devices were merely both $1.13 \%$. 
Results indicated that the $M L D$ could meet the demands of material mechanical testing machine. It was feasible to perform material mechanical experiments via parallel mechanism.

\section{Acknowledgements}

All of the experiments described herein were supported by the AVIC industry-academy-research cooperation project (CXY2013BH05).

\section{References}

[1] F.Y. Zhou, Z.Y. Mao, Engineering materials and application, second ed., Huazhong University of science and technology, Wuhan, (2002).

[2] Z.H. Shan, Mechanics of material, third ed., Higher education press, Beijing, (2009).

[3] W.M. Lu, Advanced strength and applied stress analysis., Tsinghua university, Beijing, (2001).

[4] G.P.Z, Experimental foundation of material mechanics, first ed., Harbin institute technology, Harbin, (2010).

[5] J.J. H, M.G. Lee, F. Barlat, Strain hardening response and modelling of EDDQ and DP780 steel sheet under non-linear strain path, Mech Mater. 64(2013) 11-26.

[6] S. Bruschi, T. Altan, Testing and modelling of material behaviour and formability in sheet metal forming, CIRP Annals. 63(2014) 727-749.

[7] M.M. Rodríguez, R.A. Vaz, A. Arias, Failure behaviour of 2024-T3 aluminium under tension-torsion conditions, J. Mech Sci Technol. 29(2015) 4657-4663.

[8] G.H. Zhao, L.B. Liu, Mechanical properties of AISI 1045 steel subjected to combined loads of tension and torsion, Exp Tech. 42 (2018) 393-406.

[9] J.Z. Guo, D. Wang, Development of a material testing machine with multi-dimensional loading capability, JSME. 10(2016) JAMDSM0017-JAMDSM0017. 